\title{
Effect of non-surgical periodontal therapy on glycemic control of type 2 diabetes mellitus: a systematic review and Bayesian network meta-analysis
}

Ruoyan Cao ${ }^{1}$, Qiulan $\mathrm{Li}^{2}$, Qiqi Wu${ }^{3}$, Mianfeng Yao ${ }^{4}$, Yu Chen ${ }^{1}$ and Hongbo Zhou ${ }^{{ }^{*}}$

\begin{abstract}
Background: Glycemic control is vital in the care of type 2 diabetes mellitus (T2DM) and is significantly associated with the incidence of clinical complications. This Bayesian network analysis was conducted with an aim of evaluating the efficacy of scaling and root planning (SRP) and SRP + adjuvant treatments in improving glycemic control in chronic periodontitis (CP) and T2DM patients, and to guide clinical practice.

Methods: We searched the Pubmed, Embase, Cochrane Library and Web of Science databases up to 4 May 2018 for randomized controlled trials (RCTs). This was at least three months of the duration of study that involved patients with periodontitis and T2DM without other systemic diseases given SRP. Patients in the control group did not receive treatment or SRP combination with adjuvant therapy. Outcomes were given as $\mathrm{HbA} 1 \mathrm{c} \%$ and levels fasting plasma glucose (FPG). Random-effects meta-analysis and Bayesian network meta-analysis were conducted to pool RCT data. Cochrane's risk of bias tool was used to assess the risk of bias.

Results: Fourteen RCTs were included. Most were unclear or with high risk of bias. Compared to patients who did not receive treatment, patients who received periodontal treatments showed improved $\mathrm{HbA} 1 \mathrm{c} \%$ level, including SRP (the mean difference (MD) $-0.39995 \%$ Crl 0.088 to 0.79), SRP + antibiotic (MD 0.62, 95\% Crl 0.18 to 1.11), SRP + photodynamic therapy (aPDT) + doxycycline (Doxy) (MD 1.082 95\% Crl 0.13 to 2.077) and SRP + laser (MD 0.66 95\% $\mathrm{Crl} 0.1037,1.33)$. Among the different treatments, SRP + aPDT + Doxy ranked best. Regarding fasting plasma glucose (FPG), SRP did not show advantage over no treatment (MD $4.9195 \% \mathrm{Cl}-1.95$ to 11.78) and SRP with adjuvant treatments were not better than SRP alone (MD -0.28 95\% Cl -8.66, 8.11).

Conclusion: The results of this meta-analysis seem to support that periodontal treatment with aPDT + Doxy possesses the best efficacy in lowering $\mathrm{HbA1} \mathrm{c} \%$ of non-smoking CP without severe T2DM complications. However, longer-term well-executed, multi-center trails are required to corroborate the results.
\end{abstract}

Keywords: Type 2 diabetes mellitus, Periodontitis, Non-surgical periodontal therapy, Scaling and root planing, Adjuvant therapy

\footnotetext{
* Correspondence: zhb2540@csu.edu.cn

${ }^{1}$ Department of Prosthodontics, Xiangya Stomatological Hospital \& School of

Stomatology, Central South University, 72 Xiangya Road, Changsha 410000,

China

Full list of author information is available at the end of the article
}

(c) The Author(s). 2019 Open Access This article is distributed under the terms of the Creative Commons Attribution 4.0 International License (http://creativecommons.org/licenses/by/4.0/), which permits unrestricted use, distribution, and reproduction in any medium, provided you give appropriate credit to the original author(s) and the source, provide a link to the Creative Commons license, and indicate if changes were made. The Creative Commons Public Domain Dedication waiver (http://creativecommons.org/publicdomain/zero/1.0/) applies to the data made available in this article, unless otherwise stated. 


\section{Background}

Periodontitis is a chronic inflammatory disease caused by pathogens in the surrounding periodontal tissues, that results in the periodontal pocket formation, clinical attachment loss and alveolar bone resorption and ultimately leads to tooth loss [1,2]. Epidemiological evidence has shown that periodontitis affects over $50 \%$ of the adult worldwide, indicating a dose-response relationship with oral health relates to the quality of life [3, 4]. It is well-known that periodontitis is highly associated with T2DM, and now periodontitis is regarded as the sixth complicated form of T2DM [5]. Compared to nondiabetics, patients with diabetes present worse clinical manifestation of periodontitis [6]. Besides, moderate to severe periodontitis increases the risk of T2DM and leads to poor glycemic control in diabetics $[7,8]$.

Glycemic control is vital in the care of T2DM and is significantly associated with the incidence of clinical complications. One percent reduction in $\mathrm{HbA} 1 \mathrm{c} \%$ is associated with $14 \%$ reductions in risk of myocardial infarction, $21 \%$ for deaths related to diabetes and $37 \%$ for microvascular complications [9]. Studies indicate that scaling and root planing (SRP) or SRP plus adjuvant treatment could improve glycemic control in patients with T2DM and CP, but no effective conclusion has been reached regarding the best treatment. Besides, there is conflicting evidence regarding glycemic control of various adjuvant treatments in SRP. For instance, SRP followed by locally delivered Atorvastatin (ATV) did not show a reduction of $\mathrm{HbA} 1 \mathrm{c} \%$ compared to SRP [10], while additional benefits were found after adjuvant therapy with aPDT [11]. Previous meta-analyses usually compared periodontal treatment with no treatment [12]. Few of meta-analyses have focused on adjuvant therapy, but adjuvant therapy is limited to a single type, such as aPDT [13] and systemic antibiotics [14, 15]. Therefore, a more comprehensive study is needed to clarify whether SRP or SRP with adjuvant treatments could improve glycemic control in patients diagnosed with T2DM, and potentially find the best treatment to provide evidence for clinical practice.

This Bayesian network analysis aimed to address the following focused question based on the Population, Intervention, Comparison, Outcomes, Study Design (PICOS) schema: "In chronic periodontitis with T2DM, does periodontal treatment with/without adjuvant treatment compared to no treatment or periodontal treatment with adjuvant treatment compared to periodontal treatment alone, result in better glycemic control in randomized controlled clinical trials?"

\section{Methods}

This review was not registered as a priori protocol but followed the PRISMA and the PRISMA extension for
Systematic reviews and Meta-Analyses network metaanalysis, respectively.

\section{Eligibility criteria}

The studies were considered eligible for inclusion if they met the following criteria based on PICO schema:

- Participants: Adult patients (aged $\geq 30$ years) with no gender, age and career predilection diagnosed as periodontitis and T2DM

- Intervention: Comparing SRP with no treatment, or comparing SRP with SRP plus adjuvant treatment, or comparing SRP plus adjuvant therapy with different adjuvant therapies

- Outcoming: HbA1c\% and fasting plasma glucose (FPG) (mean change in parameters from baseline to follow-up visits)

- Study design: Randomized clinical trial (RCT)

- Follow up: At least three months

- Language: English

\section{Exclusion criteria}

- Split-mouth randomized controlled clinical trial

- Pregnancy and lactation

- Current smoking and smoking within the past 5 years

- Studies including subjects who had systemic conditions (except T2DM) and major complications of T2DM

- Periodontal treatment and antibiotic use within the previous three months

- Periodontal support therapy within three months

- Sample sizes of each group less than 10

- Studies that did not report the value of HbA1c and FPG

\section{Information sources and literature search}

We searched the Pubmed, Embase, Cochrane Library and Web of Science Databases from inception to 4 May 2018. The following MeSH terms/free terms and their combinations were searched to find potentially eligible studies (Additional file 1). Also, the reference lists of relevant articles and relevant systematic reviews were manually searched to find other potentially eligible studies.

\section{Study selection}

Two independent reviewers (R.Y. Cao and Q.L. Li) screen identified eligible studies based on their titles/abstracts and full texts. Any disagreements were resolved by discussion or through an adjudication by a third review (M.F. Yao). 


\section{Data extraction and data items}

Two independent investigators (R.Y. Cao and Q.L. Li) extracted and recorded relevant data from eligible studies by pre-designed data-extraction forms: study characteristics (first author, publication year, country), patient characteristics (age, sex,inclusion criteria of T2DM and periodontitis, T2DM duration, T2DM treatment), intervention and control treatment protocols, sample size, outcome details (detection method of HbA1c\%, $\triangle \mathrm{HbA} 1 \mathrm{c} \%, \triangle \mathrm{FPG}$ ), follow-up period, adverse events. Inconsistencies were settled through discussions until an agreement was reached.

\section{Risk of bias in individual trails}

The risk of bias of included studies was assessed independently by two reviewers (R.Y. Cao and Q.L. Li) with Cochrane Collaboration tool. The items for Cochrane tool included random sequence generation; allocation concealment; blinding of participants and personnel assessment; incomplete outcome data; selective outcome reporting and other bias. We did not assess the blinding of the outcome because our outcomes (HbA1c\% and FPG) were objective indicators. Inconsistencies were settled through discussions until an agreement was reached.

\section{Outcomes and data synthesis}

The treatment outcomes were the absolute difference (AD) in $\mathrm{HbA} 1 \mathrm{c} \%$ and FPG at three to four months after periodontal treatment. When the standard deviations (SD) for the outcomes were not available, it was calculated by assuming that the correlation coefficient was 0.5 as previously described $[16,17]$.

The data used was a follow-up of 3-4 months. First, a random-effects pairwise meta-analysis was performed with the Stata 14.2 (Stata Corporation, College Station, TX, USA). The mean difference (MD) and 95\% confidence intervals (CIs) were used to compare continuous variables as previously described [12]. Second, we did a Bayesian network analysis by using Markov chain Monte Carlo methods via GeMTC package 0.8 implemented in $\mathrm{R}$ 3.2.2. We also assessed study design information and patient characteristics to evaluate the transitivity assumption for reliable data pooling with sufficient similarity between the included trials $[18,19]$. Both non-informative uniform and normal prior distributions were used throughout the network meta-analysis [20]. Markov chain Monte Carlo methods with four chains of 300,000 iterations after a burn-in phase of 180,000 iterations were used to gain MD and $95 \%$ credible intervals (CrIs). We used CrIs beyond the null value to assess significance. We also performed a sensitivity analysis by setting a uniform prior standard deviation ("std.dev", "dunif", 0, 2). Model selection (fixed vs random effects) was based on the assessment of the deviance information criterion (DIC) [21]. We then reported the results of the random-effects model. Brooks-GelmanRubin diagnostic plot was used to assess model convergence of network analysis [19]. We also ranked the different treatments in terms of lowering the HbA1c\% level using the same methods [20].

The goodness of fit of the model assessed the consistency assumption of this network analysis and it was tested by calculating the posterior mean residual deviance (Dbar). When the Dbar was similar to the number of data points in the study, the model was taken into account for fitting the data well [22]. Moreover, we also examined the pooled effects from traditional pairwise meta-analysis and network meta-analysis to further verify the consistency of the network. Inconsistency was assessed by comparing direct evidence with indirect evidence from the entire network on each node (node-splitting analysis) with $p<0.05$ [23]. Heterogeneity was assessed by $\mathrm{I}^{2}$ calculation. To verify the robustness of our analyses, we performed a sensitivity analysis by excluding studies with $\mathrm{HbA} 1 \mathrm{c} \%>9$ or $<7$ as the baseline. We also conducted comparison-adjusted funnel plots to assess publication bias. All statistical analyses were performed using Review Manager 5.3 (Cochrane Collaboration, Oxford, UK), R 3.2.2 (R Foundation for Statistical Computing, Vienna, Austria) and Stata 14.2 (Stata Corporation, College Station, TX, USA).

\section{Quality of network meta-evidence}

We assessed the quality of evidence for direct estimates based on the GRADE criteria [24] using five items (risk of bias, imprecision, indirectness, inconsistency and publication bias). We used an approach that proposed by Puhan et al. and Brignardello-Petersen et al. to assess the quality of evidence for indirect and network estimates.

\section{Results \\ Study selection}

The literature search identified 586 possibly eligible articles, 86 articles were fully accessed after excluding duplicates and unsuitable studies by title/abstract, while 72 of them were not considered eligible for inclusion (Additional file 2). A total of 14 trials with 629 patients were included in this network meta-analysis. Figure 1 shows phases of the screening process.

\section{Characteristics of included studies}

Tables 1 and 2 presented the main characteristics of the 14 included studies. All studies were RCTs, and were followed up for 3-12 months. All the patients had T2DM and chronic periodontitis, but the diagnostic criteria of T2DM were not reported in most studies. The T2DM duration was from 4 to 11.8 years. T2DM treatments mainly included diet and insulin supplementation 


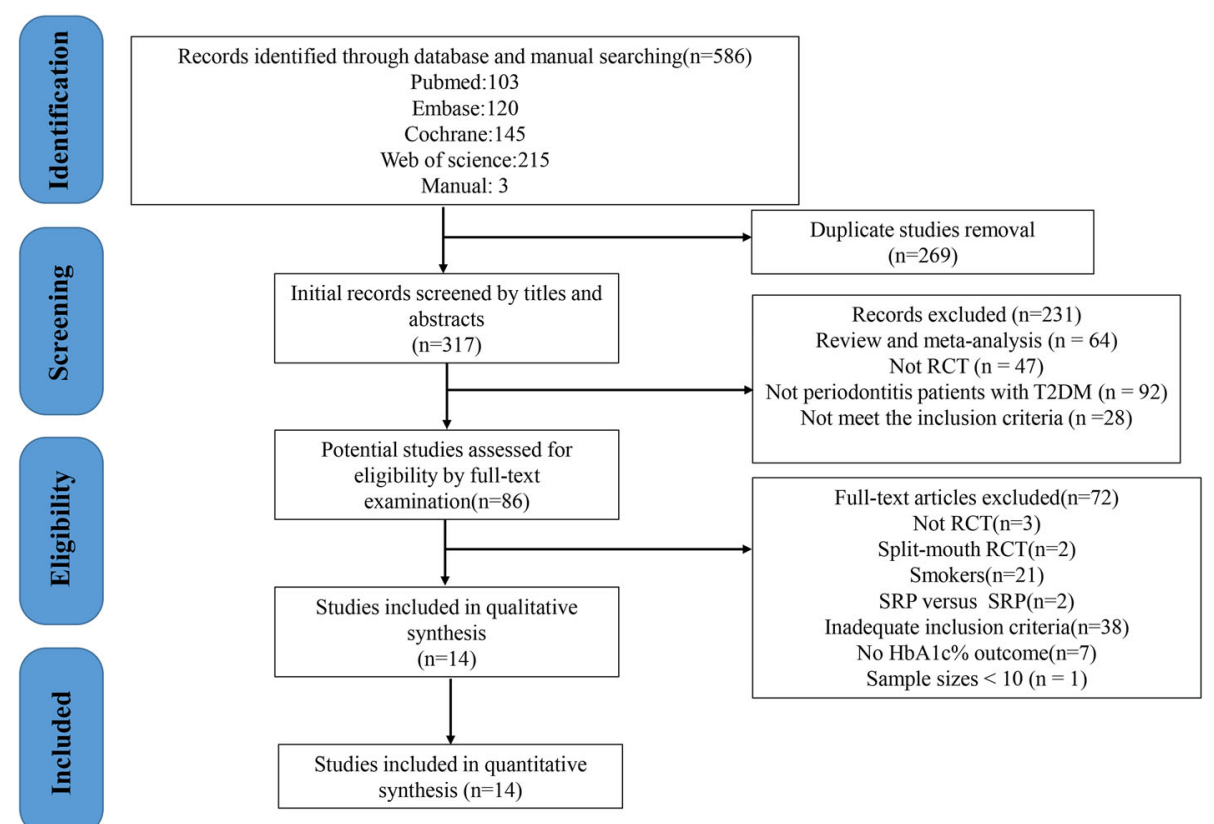

Fig. 1 Flow chart of articles search and screening process

or oral hypoglycemic agents. The baseline of HbA1c\% varied widely, from 6.2 to 10.4. Periodontal treatments were performed by SRP, within $24 \mathrm{~h}$ - 2 weeks. Most studies gave oral hygiene instruction to subjects. All studies reported the outcomes of $\mathrm{HbA1c} \%$, few reported on FPG. Four studies did not report adverse events, and three studies [30, 32, 33] showed side effects, such as taste perception, dry mouth, headache, diarrhoea. Ten studies reported the method of HbA1c\% determination. The network meta-analysis included 7 treatments (Fig. 2), including SRP $(n=$ $280)$, no treatment $(n=76)$, SRP + antibiotic (doxycycline (Doxy), metronidazole + amoxicillin, Doxy) $(n=$ 130), subantimicrobial dose doxycycline (SDD) $(n=17)$, locally-delivered drugs (atorvastatin gel, simvastatin gel, chlorhexidine gel) $(n=66)$, laser (diode laser, aPDT) $(n=$ $45)$ and SRP + Doxy + aPDT $(n=15)$. The plots indicated that most of the direct evidence between different adjuvantive treatments was lacking.

\section{Risk of bias in included studies}

Figure 3 presented reviewers' judgments on the risk of bias in the referred RCTs. Most included studies were found to have methodological issues, with the most problematic domains being the allocation concealment (unclear or high risk in $35.7 \%$ of studies). Though the included studies were RCTs, $35.7 \%$ studies did not report the details of the randomized method. One study had an low risk of selective outcome reporting bias. In addition, two studies also might have had other bias, because we could not judge if there were baseline imbalances between groups (Additional file 3).

\section{Efficacy of different treatments for the reduction of $\mathrm{HbA} 1 \mathrm{c} \%$ in subjects with $\mathrm{CP}$ and T2DM}

In traditional meta-analysis (Table 3), SRP + laser showed additional benefits compared to SRP alone $(0.19$ $[0.08,0.30])$. There was statistically significant reduction in $\mathrm{HbA} 1 \mathrm{c} \%$ after treated with SRP + antibiotic (0.82 $[0.33,1.31])$ compared to no treatment. In addition, there was no other significant difference was observed among the rest of the comparisons. The overall heterogeneity was high (Global $\left.\mathrm{I}^{2}=83.2\right)$, but mainly between SRP and no treatment $\left(I^{2}=94.2\right)$, while the heterogeneity was lower in other subgroups $\left(\mathrm{I}^{2}=0\right)$.

In network meta-analysis, sensitivity analysis indicated that both non-informative uniform and normal prior distributions were properly used (Additional file 4). SRP + antibiotic $(0.61[0.16,1.1])$, SRP + aPDT + Doxy (1.1 $[0.11,2.1])$, SRP + laser $(0.66[0.097,1.3])$ and SRP alone $(0.40[0.086,0.80])$ was significantly better than no treatment in improving $\mathrm{HbA} 1 \mathrm{c} \%$, separately (Fig. 4). No other significant difference was observed among the rest of the comparisons. The probabilities the most effective treatment methods of decreasing $\mathrm{HbA} 1 \mathrm{c} \%$ was SRP + aPDT + Doxy (71.2\%), followed by SRP + laser (13.6\%), SRP + SDD (8.6\%), SRP + antibiotic (3.8\%), SRP (0.2\%) and no treatment $(0.02 \%)$ (Fig. 5). The Global $\mathrm{I}^{2}$ was 86.18, except that between SRP and no treatment or SRP + laser were 95.63 and 23.56, respectively, the other subgroups were 0 (Table 4 ). 


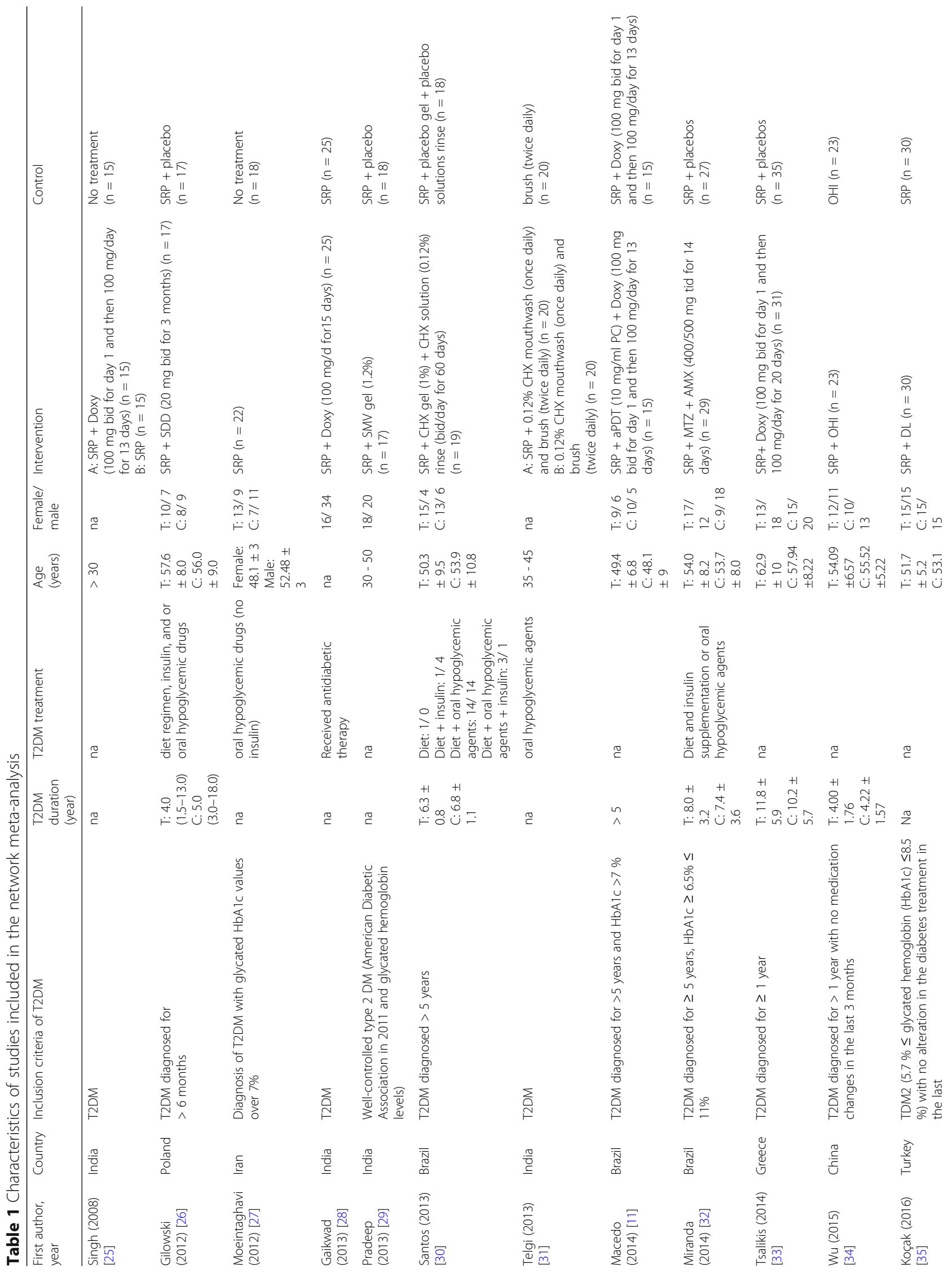




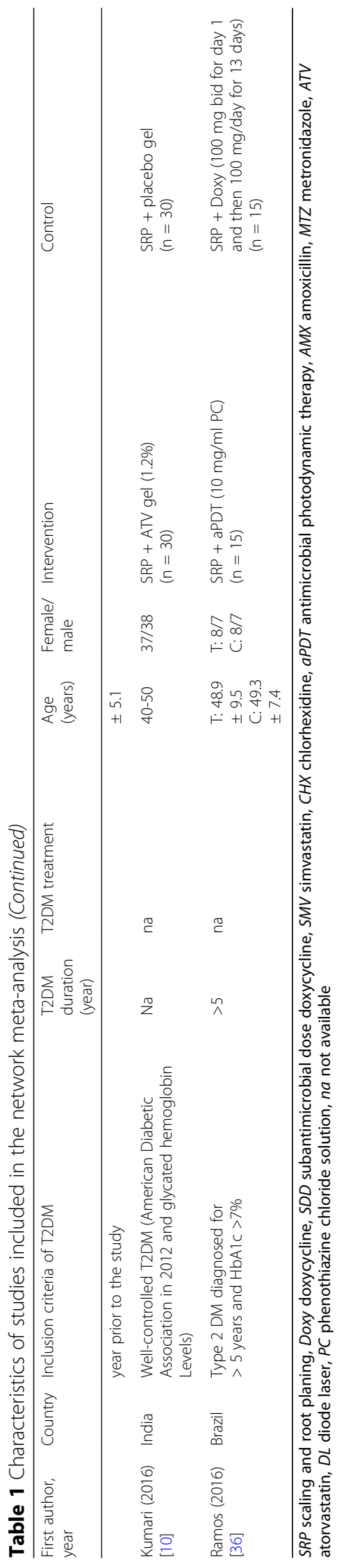


Cao et al. BMC Oral Health

(2019) 19:176

Page 7 of 14

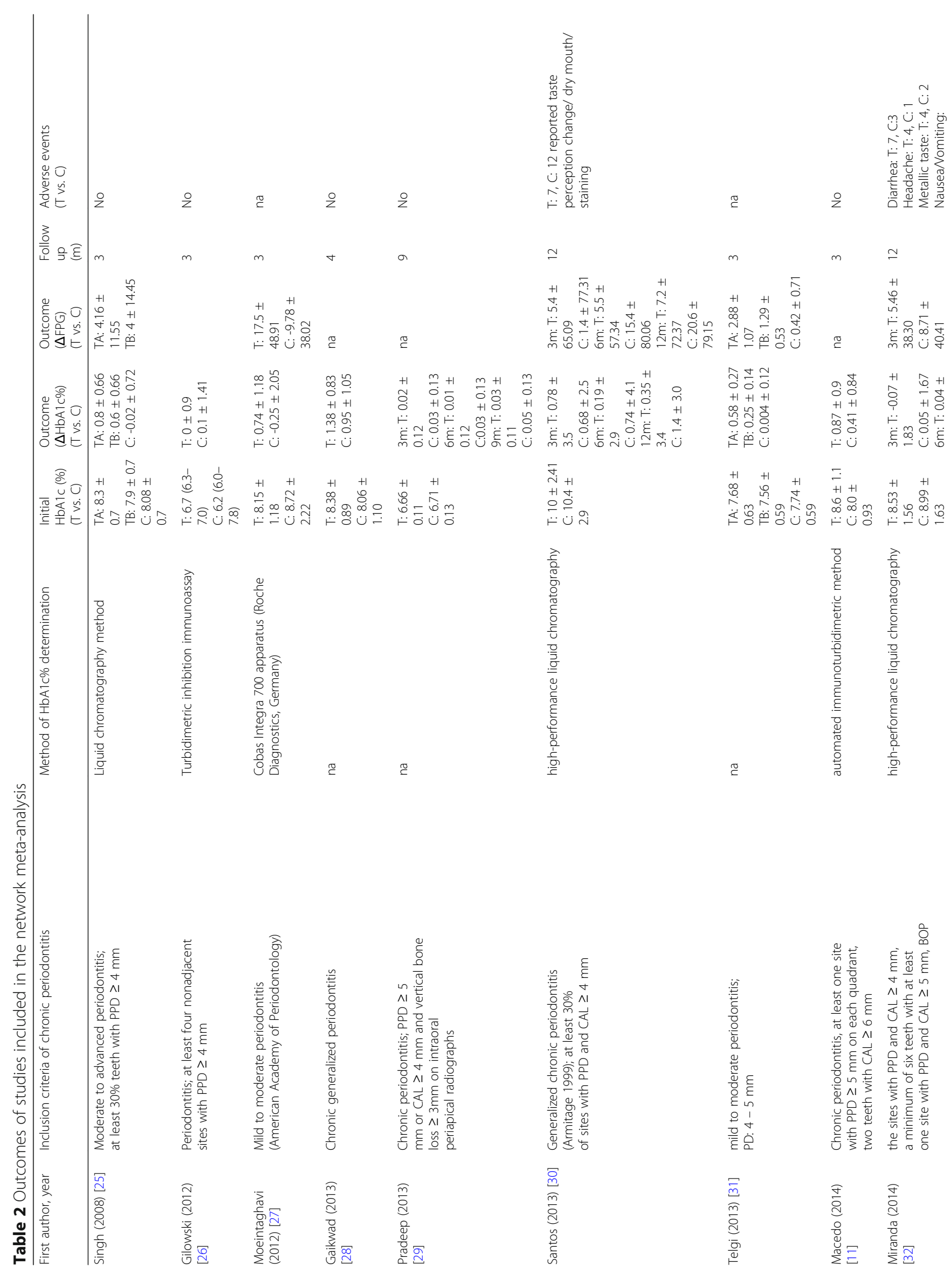




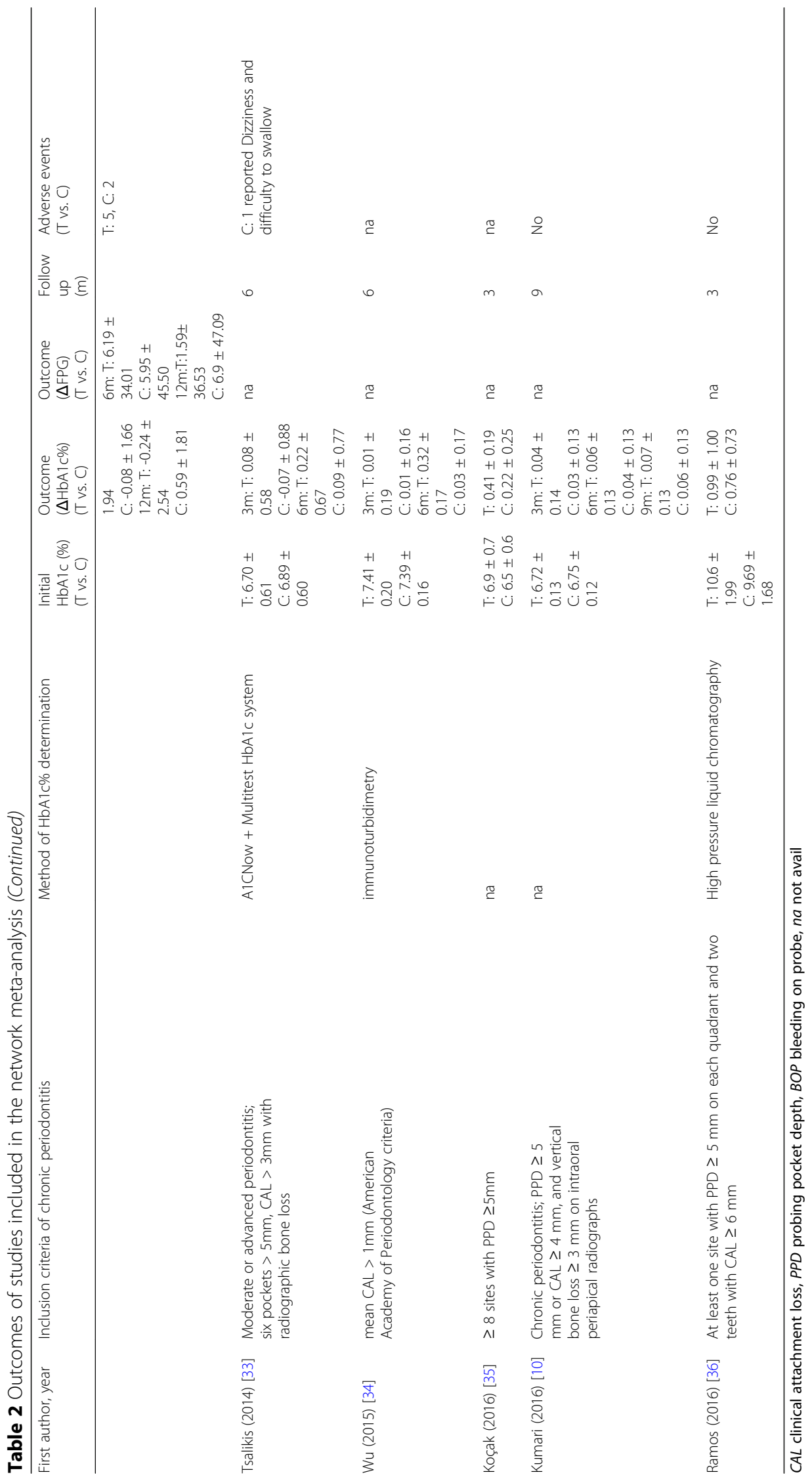




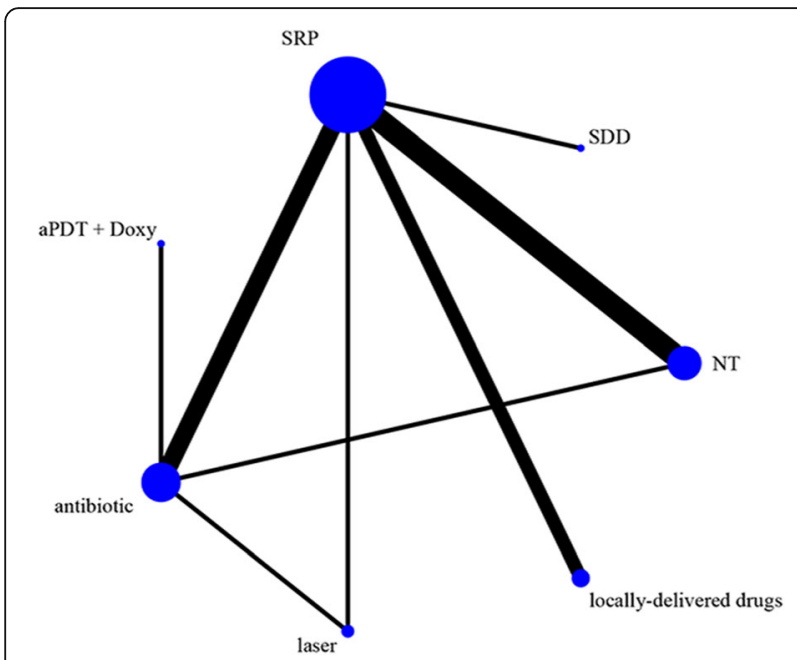

Fig. 2 Network of the interventional comparisons for the Bayesian network analysis. The size of the nodes is proportional to the number of subjects (sample size) randomized to receive the therapy. The width of the lines is proportional to the number of trials comparing each pair of treatments. aPDT, antimicrobial photodynamic therapy; Doxy, doxycycline; antibiotics (Doxy, metronidazole + amoxicillin); local, locally-delivered drugs (atorvastatin gel, chlorhexidine gel, simvastatin gel); laser (diode laser, aPDT); SDD, subantimicrobial dose doxycycline; SRP, scaling and root planing, NT, no treatment

Efficacy of different treatments for the reduction of FPG in subjects with CP and T2DM

We only did a pairwise meta-analysis, because there were only 5 available studies. SRP was not better than no treatment in FPG reduction $\left(4.91\right.$ [- 1.95, 11.78], $\mathrm{I}^{2}=$ 46.6\%) (Additional file 5). Moreover, no significant difference was observed between SRP + adjuvant and SRP $\left(-0.28[-8.66,8.11], \mathrm{I}^{2}=0.0 \%\right)$.

\section{Model fit and evaluation of consistency}

The model fitted the data well for the Dbar approximated data points in both outcomes (Additional file 6), and the effects of most comparisons between pairwise and network meta-analysis were similar in the relevant CI or CrI (Table 3). In addition, the node-splitting analysis also showed that there was no inconsistency among direct, indirect and network outcomes with $P>0.05$ (Table 5).

\section{Sensitivity analysis and publication bias}

To explore whether the baseline level affects the final effects, we excluded studies with relative higher $\mathrm{HbA} 1 \mathrm{c} \%$ (>9\%) or relative lower $\mathrm{HbA1c} \%(<7 \%)$. The results were shown in Additional file 7. When we excluded the studies with $\mathrm{HbA} 1 \mathrm{c} \%>9 \%$, no major changes were found except for SRP + laser versus no treatment, from $0.66[0.097,1.3]$ to $0.59[-0.15,1.4]$. Nevertheless, if we excluded studies with $\mathrm{HbA} 1 \mathrm{c} \%<7 \%$, no significant

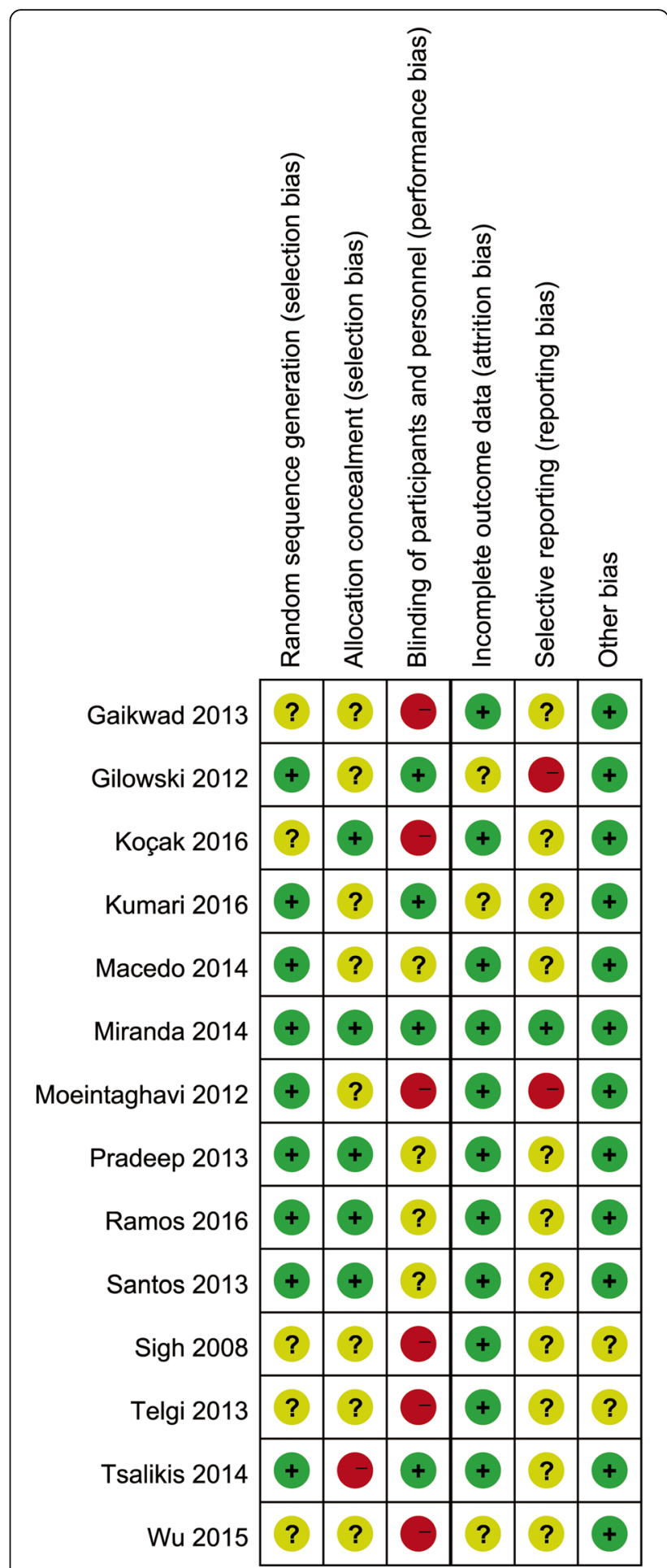

Fig. 3 Judgements about each risk of bias item for each included study

difference was found any treatment comparisons. The result of the comparison-adjusted funnel plot of $\mathrm{HbA} 1 \mathrm{c} \%$ reduction revealed there may be publication bias owing asymmetry (Fig. 6). 
Table 3 Comparison of outcomes between traditional meta-analysis and Bayesian network meta-analysis

\begin{tabular}{lll}
\hline treatment comparison & traditional meta-analysis & network meta-analysis \\
\hline SRP + Antibiotic vs. SRP & $0.21(-0.03,0.45)$ & $0.20(-0.17,0.56)$ \\
SRP + Laser vs. SRP & $0.19(0.08,0.30)$ & $0.25(-0.24,0.78)$ \\
SRP + locally-delivered drug vs. SRP & $0.00(-0.05,0.05)$ & $0.0026(-0.41,0.43)$ \\
SRP + SDD vs. SRP & $-0.10(-0.90,0.70)$ & $-0.10(-1.1,0.89)$ \\
SRP + aPDT + Doxy vs. SRP + Antibiotic & $0.46(-0.16,1.08)$ & $0.46(-0.40,1.31)$ \\
SRP + Laser vs. SRP + Antibiotic & $0.23(-0.40,0.86)$ & $0.053(-0.48,0.61)$ \\
SRP vs. No treatment & $0.45(0.00,0.89)$ & $0.40(0.088,0.80)$ \\
SRP + Antibiotic vs. No treatment & $0.82(0.33,1.31)$ & $0.61(0.16,1.11)$ \\
\hline
\end{tabular}

SRP scaling and root planing, SDD subantimicrobial dose doxycycline, aPDT antimicrobial photodynamic therapy, Doxy doxycycline

\section{Quality of network meta-evidence}

We used Grade approach to assess the quality of evidence and the results were presented in Fig. 4. Most of the comparisons were found to be of low quality evidence, four comparisons (SRP + antibiotic vs. SRP + aPDT + Doxy, SRP + laser vs. SRP/SRP + antibiotic/ aPDT + Doxy) were considered to have moderate quality of evidence and three comparisons (SRP + laser/ local-delivery drugs) were considered as lowquality of evidence. These indicate that we have limited confidence in these recommendations, and future research may change them.

\section{Discussion}

This network meta-analysis of 14 RCTs including 629 patients that compared the $\mathrm{HbA} 1 \mathrm{c} \%$ reduction of different treatments indicated that all the treatments might improve HcA1c\% than no treatment except for SDD or local-delivery drugs adjunctive to SRP. Furthermore, among the treatments, SRP + aPDT + Doxy was the most effective. No evidence of effectiveness was found in the lowering of FPG.
Evidence-based data of the periodontal treatment effect on glycemic control in T2DM are limited. Although some studies have compared SRP with no treatment or SRP + adjuvant treatment, there was still a lack of sufficient data comparing the different adjuvant treatments. Our study included all periodontal treatments that met our inclusion criteria and made a more overall evaluation. The results from network meta-analysis and traditional meta-analysis were largely consistent. Even so, laser might improve the efficacy of SRP in $\mathrm{HbA} 1 \mathrm{c} \%$ reduction in our pairwise meta-analysis $(0.19[0.08,0.30])$, but not in our network meta-analysis $(0.25[-0.24$, 0.78]). This inconsistency may be attributed to chance alone, as only one study with small sample size provided direct evidence. The inconsistency was also found when comparing SRP with no treatment. SRP could reduce periodontal pathogens, and subsequently inhibit cytokines associated with inflammatory markers, leading to decreased glucose [37]. Our network meta-analysis supported the efficacy of SRP $(0.40[0.088,0.80])$, and was consistent with previous meta-analyses $[5,38]$. Although our pairwise meta-analysis showed that SRP was not better than no treatment $(0.45[0.00,0.89])$, the lower

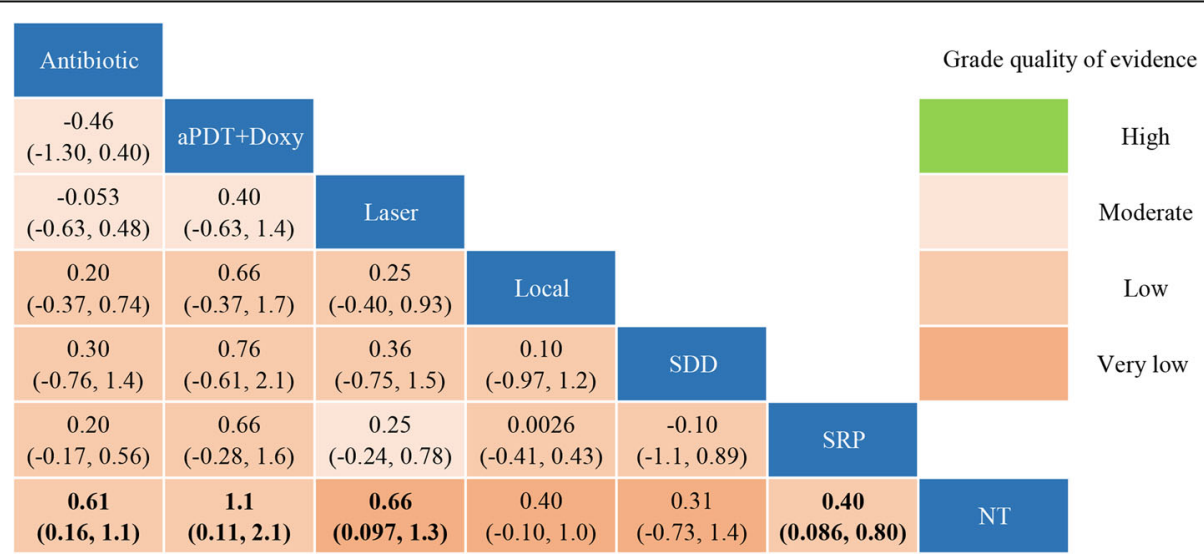

Fig. 4 Multiple-treatment comparisons and the quality of evidence for $\Delta \mathrm{HbAlc} \%$. aPDT, antimicrobial photodynamic therapy; Doxy, doxycycline; antibiotics (Doxy, metronidazole + amoxicillin); local, locally-delivered drugs (atorvastatin gel, chlorhexidine gel, simvastatin gel); laser (diode laser, aPDT); SDD, subantimicrobial dose doxycycline; SRP, scaling and root planing, NT, no treatment 


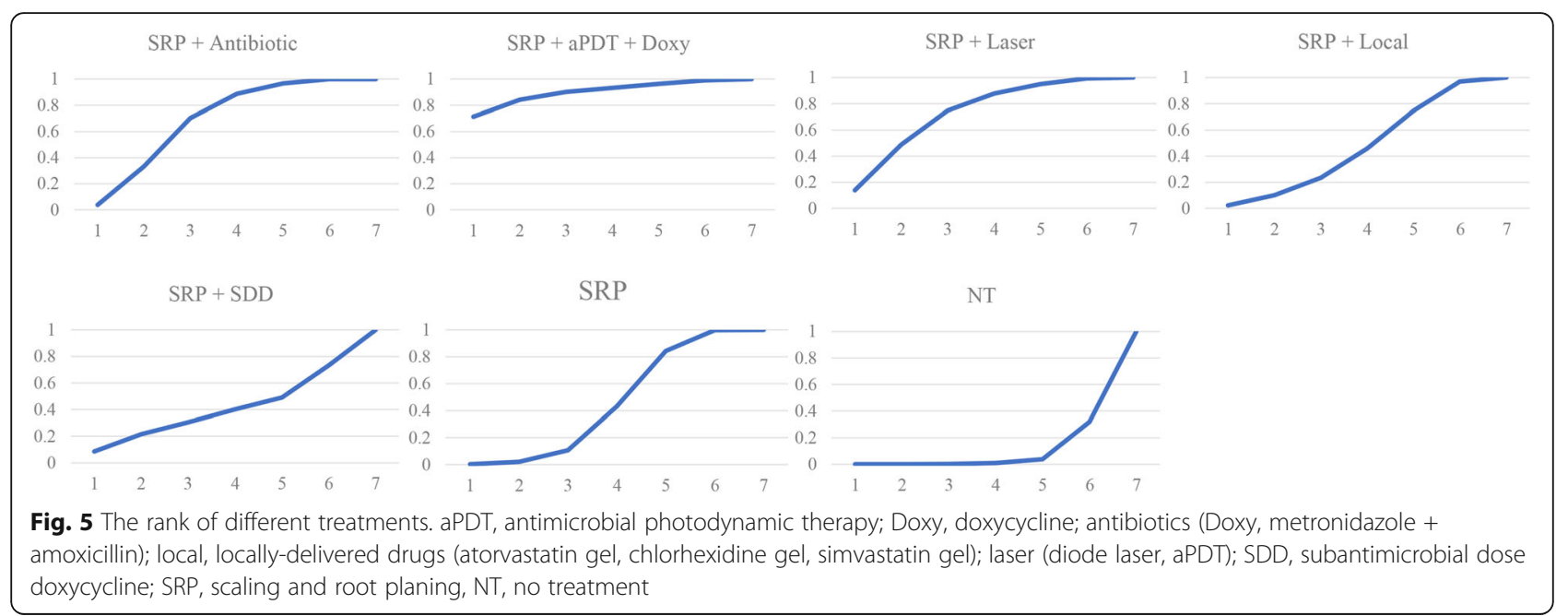

bound of the $95 \%$ CI was 0. Heterogeneity between SRP and no treatment is extremely high in both pairwise and network meta-analysis, this may due to the fact that the baseline of $\mathrm{HbA1c} \%$ is not balanced in the 3 included studies and they may have serious methodological issues. For example, they did not report how to perform allocation concealment. More well-executed and multi-center trails are still required to explore the effect of SRP.

We also found an interesting phenomenon that SRP + SDD / local-delivery drugs did not showed any advantages of improving $\mathrm{HbA} 1 \mathrm{c} \%$ than no treatment. It seems illogical since SRP alone was better than no treatment. There was only one study with small sample sizes in SRP + SDD; further trials are needed to ascertain the role of SDD in improving $\mathrm{HbA} 1 \mathrm{c} \%$. The uncomparable baseline of $\mathrm{HbA} 1 \mathrm{c} \%$ may be attributed to the inefficacy of SRP + local-delivery drugs: HbA1c\% $>10$ for Santos et al. [30]; HbA1c\% $<7$ for Pradeep et al. [29], Kumari et al.
Sensitivity analyses indicated that the baseline of HbA1c\% may influence the efficacy of the periodontal treatments. After we excluded the studies with $\mathrm{HbA1c} \%$ > 9, the major results did not change greatly. Nonetheless, when the studies with $\mathrm{HbA} 1 \mathrm{c} \%<7$ were excluded, all treatments except SRP + antibiotic could not significantly decrease $\mathrm{HbA} 1 \mathrm{c} \%$ compared to no treatment. Longitudinal studies involving subjects with different $\mathrm{HbA} 1 \mathrm{c} \%$ is therefore warranted.

Antibiotics are commonly applied in periodontal treatment. They could significantly decrease the levels of TNF and IL-6 in serum [39]. TNF and IL-6 could impair intracellular insulin signalling, potentially leading to insulin resistance [40]. Contrary to our expectations, we found it couldn't provide additional benefits compared to SRP alone $(0.20[-0.17,0.56])$. This was in conformance with findings of Wang et al. $(-0.238[-0.616,0.140])[11]$ and Lira Junior et al. $(-0.11[-0.35,0.13])[14]$.

Table 4 Analysis of heterogeneity

\begin{tabular}{|c|c|c|c|c|}
\hline t1 & t2 & i2.pair & i2.cons & incons.p \\
\hline \multicolumn{5}{|l|}{$\mathrm{HbA} 1 \mathrm{c} \%$} \\
\hline \multicolumn{5}{|l|}{ Per-comparison I-squared } \\
\hline SRP + Antibiotic & $\mathrm{SRP}+\mathrm{aPDT}+$ Doxy & NA & NA & NA \\
\hline SRP + Antibiotic & SRP + Laser & NA & 0.00 & 0.62 \\
\hline SRP + Antibiotic & NT & NA & 0.00 & 0.587 \\
\hline SRP + Antibiotic & SRP & 0.00 & 0.00 & 0.98 \\
\hline $\mathrm{SRP}+\mathrm{SDD}$ & SRP & NA & NA & NA \\
\hline SRP + Laser & SRP & NA & 23.56 & 0.77 \\
\hline SRP + Locally-delivered drugs & SRP & 0.00 & 0.00 & NA \\
\hline NT & SRP & 95.43 & 95.63 & NA \\
\hline Global I-squared & & 88.11 & 86.18 & \\
\hline
\end{tabular}

$S R P$ scaling and root planing, SDD subantimicrobial dose doxycycline, $t 1$ treatment $1, t 2$ treatment 2 , i2.pair i-square of pair-wise meta-analysis, i2.cons i-square of network meta-analysis, incons.p inconsistency p-values for pairwise and network meta-analysis, NA not applicable 
Table $\mathbf{5}$ The results of node-spitting analysis

\begin{tabular}{|c|c|c|}
\hline comparison & $p$-value & $\mathrm{MD}(95 \% \mathrm{Crl})$ \\
\hline \multicolumn{3}{|l|}{$\mathrm{HbA} 1 \mathrm{c} \%$} \\
\hline \multicolumn{3}{|c|}{ SRP + Antibiotic vs. SRP + Laser } \\
\hline direct & 0.61455 & $0.23(-0.66,1.1)$ \\
\hline indirect & & $-0.059(-0.83,0.72)$ \\
\hline network & & $0.043(-0.49,0.62)$ \\
\hline \multicolumn{3}{|c|}{ SRP + Antibiotic vs. No treatment } \\
\hline direct & 0.5605667 & $-0.82(-1.7,0.036)$ \\
\hline indirect & & $-0.53(-1.2,0.090)$ \\
\hline network & & $-0.62(-1.1,-0.18)$ \\
\hline \multicolumn{3}{|c|}{ SRP + Antibiotic vs. SRP } \\
\hline direct & 0.6489667 & $-0.22(-0.65,0.22)$ \\
\hline indirect & & $0.040(-1.1,1.2)$ \\
\hline network & & $-0.22(-0.58,0.15)$ \\
\hline \multicolumn{3}{|c|}{ SRP + Laser vs. SRP } \\
\hline direct & 0.5950500 & $-0.19(-0.85,0.47)$ \\
\hline indirect & & $-0.48(-1.5,0.50)$ \\
\hline network & & $-0.26(-0.79,0.23)$ \\
\hline
\end{tabular}

Laser, especially aPDT has been attracting huge interest in adjunct to SRP due to its better antibacterial ability with lower technically sensitive. In our network meta-analysis, we included three RCTs that compared laser to other treatments (SRP + aPDT vs. SRP, SRP + diode laser vs. SRP and SRP + aPDT vs. SRP + Doxy). We found SRP + laser was better compared to no treatment $(0.66[0.097,1.3])$ but not SRP $(0.25[-0.24$, $0.78]$ ). Similarly, a meta-analysis of 2 RCTs was conducted by Abduljabbar et al. showed that aPDT didn't provide additional benefits compared to SRP alone (0.035 [- 0.47, 0.54]) [13]. However, one of its included RCT [11] was used in treating their patients with doxycycline. In our opinion, it might intensify the effect of aPDT, so we considered it as an independent group (aPDT + Doxy) rather than the laser group. In our network meta-analysis, aPDT + Doxy adjunctive to SRP was ranked best among the comparisons, although there was only one trial of 30 patients was included. It is difficult to draw a solid conclusion with such a small amount of RCTs. Thus, we look forward to more wellexecuted and multi-center trails.

Regarding FPG, SRP was not better than no treatment (4.91 [-1.95, 11.78]), and adjuvant treatments did not show any advantage than SRP alone $(-0.28[-8.66$, 8.11]). The results are consistent with Corbella et al. [17]. However, Sgolastra et al. [41] and Teshome et al. [42] support the effectiveness of periodontal treatment in lowering FPG. Since few studies have reported the results of FPG, it is difficult to draw a conclusion. Therefore, more RCTs were expected to clear out the real effect of periodontal treatments.

This network meta-analysis has several limitations. First, only included English studies were included and their quality is the principal limitation, a low risk of bias was found in six of the 14 included studies. Second, the sample sizes were relatively small, from 30 to 66 subjects. The follow-up duration of the trials was short,

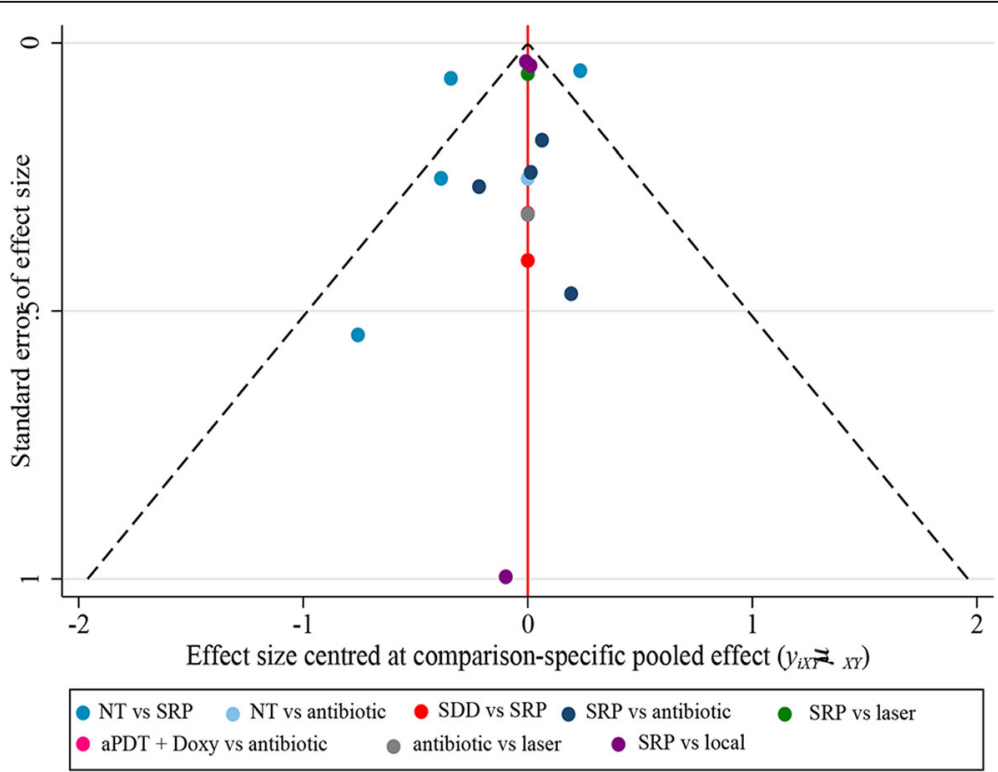

Fig. 6 Publication bias assessment for $\Delta H b$ Alc\%. aPDT, antimicrobial photodynamic therapy; Doxy, doxycycline; antibiotics (Doxy, metronidazole + amoxicillin); local, locally-delivered drugs (atorvastatin gel, chlorhexidine gel, simvastatin gel); laser (diode laser, aPDT); SDD, subantimicrobial dose doxycycline; SRP, scaling and root planing, NT, no treatment 
resulting in uncertain outcomes. With the foregoing limitations, well-executed and multi-center trails comparing the different adjuvant treatments, and extending the follow-up duration up to 12 or 24 months should be conducted. Finally, this meta-analysis excluded splitmouth RCT, patients with severe T2DM complications and smorkers to meet the transitivity, indicating that the conclusions of this meta-analysis apply to non-smoking CP patients without severe T2DM complications. In addition, though we have adopted strict eligibility criteria, we also found high heterogeneity in comparing SRP with no treatment. We also found a certain publication bias.

\section{Conclusion}

The results of this meta-analysis seem to support that periodontal treatment with aPDT + Doxy possesses the best efficacy in lowering $\mathrm{HbA1c} \%$ of non-smoking $\mathrm{CP}$ without severe T2DM complications. However, the quality of evidence is low or very low, and therefore further studies are needed to confirm the results.

\section{Additional files}

\section{Additional file 1: Search strategy used in PubMed/MEDLINE. (DOCX $14 \mathrm{~kb}$ )}

Additional file 2: List of excluded duplicate studies. (DOCX $32 \mathrm{~kb}$ )

Additional file 3: Sensitivity analysis for informative uniform distribution. (DOCX $24 \mathrm{~kb}$ )

Additional file 4: Risk of bias summary: review authors' judgements about each risk of bias item for each included study. (DOCX 342 kb)

Additional file 5: Forest plot of changes in FPG. (DOCX $172 \mathrm{~kb}$ )

Additional file 6: Evaluation of model fit. (DOCX $14 \mathrm{~kb}$ )

Additional file 7: Sensitivity analysis. (DOCX $16 \mathrm{~kb}$ )

Additional file 8: Data extraction table of this network meta-analysis. (DOCX $16 \mathrm{~kb}$ )

\section{Abbreviations}

CP: Chronic periodontitis; T2DM: Type 2 diabetes mellitus; SRP: Scaling and root planning; Doxy: Doxycycline; SDD: Subantimicrobial dose doxycycline; SMV: Simvastatin; CHX: Chlorhexidine; aPDT: Antimicrobial photodynamic therapy; AMX: Amoxicillin; MTZ: Metronidazole; ATV: Atorvastatin; DL: Diode laser; PC: Phenothiazine chloride solution

\section{Acknowledgements}

We want to acknowledge Dr. Jianying Zhang (Central South University) for her help during this manuscript development.

\section{Authors' contributions}

RYC, QLL and MFY were responsible for study selection, quality assessment, data extraction and data synthesis. RYC drafted the manuscript. HBZ, QQW and $Y C$ participated in the research design and revision of the manuscript. All authors read and approved the final manuscript.

\section{Funding}

There was no funding for this review.

\section{Availability of data and materials}

All data generated or analysed during this study are included in this published article and Additional file 8.
Ethics approval and consent to participate

Not applicable.

\section{Consent for publication}

Not applicable.

\section{Competing interests}

The authors declare that they have no competing interests.

\section{Author details}

${ }^{1}$ Department of Prosthodontics, Xiangya Stomatological Hospital \& School of Stomatology, Central South University, 72 Xiangya Road, Changsha 410000, China. 'Department of Stomatology, The Second Xiangya Hospital, Central South University, 139 Middle Renmin Road, Changsha 410011, China.

${ }^{3}$ Department of Operative Dentistry and Endodontics, Xiangya Stomatological Hospital \& School of Stomatology, Central South University, 72 Xiangya Road, Changsha 410000, China. ${ }^{4}$ Department of Oral Medicine, Xiangya Hospital, Central South University, 87 Xiangya Road, Changsha 410083, China.

Received: 9 July 2018 Accepted: 23 June 2019

Published online: 06 August 2019

\section{References}

1. Kinane DF, Stathopoulou PG, Papapanou PN. Periodontal diseases. Nat Rev Dis Primers. 2017;3:17038.

2. Zhang C, Lu Y, Zhang L, Liu Y, Zhou Y, Chen Y, Yu H. Influence of different intensities of vibration on proliferation and differentiation of human periodontal ligament stem cells. Arch Med Sci. 2015:11(3):638-46.

3. Buset SL, Walter C, Friedmann A, Weiger R, Borgnakke WS, Zitzmann NU. Are periodontal diseases really silent? A systematic review of their effect on quality of life. J Clin Periodontol. 2016;43(4):333-44.

4. Tonetti MS, Eickholz P, Loos BG, Papapanou P, van der Velden U, Armitage G, Bouchard P, Deinzer R, Dietrich T, Hughes F, et al. Principles in prevention of periodontal diseases: consensus report of group 1 of the 11th European workshop on periodontology on effective prevention of periodontal and peri-implant diseases. J Clin Periodontol. 2015;42(Suppl 16):S5-11.

5. Mizuno H, Ekuni D, Maruyama T, Kataoka K, Yoneda T, Fukuhara D, Sugiura Y, Tomofuji T, Wada J, Morita M. The effects of non-surgical periodontal treatment on glycemic control, oxidative stress balance and quality of life in patients with type 2 diabetes: a randomized clinical trial. PLoS One. 2017; 12(11):e0188171.

6. Sun X, Mao Y, Dai P, Li X, Gu W, Wang H, Wu G, Ma J, Huang S. Mitochondrial dysfunction is involved in the aggravation of periodontitis by diabetes. J Clin Periodontol. 2017:44(5):463-71.

7. Winning L, Patterson CC, Neville CE, Kee F, Linden GJ. Periodontitis and incident type 2 diabetes: a prospective cohort study. J Clin Periodontol. 2017:44(3):266-74

8. Zhou X, Zhang W, Liu X, Zhang W, Li Y. Interrelationship between diabetes and periodontitis: role of hyperlipidemia. Arch Oral Biol. 2015;60(4):667-74.

9. Stratton IM, Adler Al, Neil HA, Matthews DR, Manley SE, Cull CA, Hadden D, Turner RC, Holman RR. Association of glycaemia with macrovascular and microvascular complications of type 2 diabetes (UKPDS 35): prospective observational study. BMJ. 2000:321(7258):405-12.

10. Kumari M, Martande SS, Pradeep AR, Naik SB. Efficacy of Subgingivally delivered $1.2 \%$ atorvastatin in the treatment of chronic periodontitis in patients with type 2 diabetes mellitus: a randomized controlled clinical trial. J Periodontol. 2016:87(11):1278-85.

11. Macedo Gde O, Novaes AB Jr, Souza SL, Taba M Jr, Palioto DB, Grisi MF. Additional effects of aPDT on nonsurgical periodontal treatment with doxycycline in type II diabetes: a randomized, controlled clinical trial. Lasers Med Sci. 2014;29(3):881-6.

12. Simpson TC, Weldon JC, Worthington HV, Needleman I, Wild SH, Moles DR, Stevenson B, Furness S, Iheozor-Ejiofor Z. Treatment of periodontal disease for glycaemic control in people with diabetes mellitus. Cochrane Database Syst Rev. 2015;(11):CD004714.

13. Abduljabbar T, Vohra F, Javed F, Akram Z. Antimicrobial photodynamic therapy adjuvant to non-surgical periodontal therapy in patients with diabetes mellitus: a meta-analysis. Photodiagn Photodyn Ther. 2017;17:138-46. 
14. Lira Junior R, Santos CMM, Oliveira BH, Fischer RG, Santos APP. Effects on $\mathrm{HbA1c}$ in diabetic patients of adjunctive use of systemic antibiotics in nonsurgical periodontal treatment: a systematic review. J Dent. 2017;66:1-7.

15. Wang TF, Jen IA, Chou C, Lei YP. Effects of periodontal therapy on metabolic control in patients with type 2 diabetes mellitus and periodontal disease: a meta-analysis. Medicine. 2014;93(28):e292.

16. Wang X, Han X, Guo X, Luo X, Wang D. The effect of periodontal treatment on hemoglobin a1c levels of diabetic patients: a systematic review and meta-analysis. PLoS One. 2014;9(9):e108412.

17. Corbella S, Francetti L, Taschieri S, De Siena F, Fabbro MD. Effect of periodontal treatment on glycemic control of patients with diabetes: a systematic review and meta-analysis. J Diabetes Investig. 2013;4(5):502-9.

18. Wang T, Wang X, Yang K, Zhang J, Luo J, Gao P, Ma Y, Jiao L, Ling F. Endovascular treatment for symptomatic intracranial artery stenosis: protocol for a systematic review and network meta-analysis. BMJ Open. 2018;8(7):e022359.

19. Yogendrakumar V, Lun R, Hutton B, Fergusson DA, Dowlatshahi D. Comparing pharmacological venous thromboembolism prophylaxis to intermittent pneumatic compression in acute intracerebral haemorrhage: protocol for a systematic review and network meta-analysis. BMJ Open. 2018:8(11):e024405.

20. Cipriani A, Furukawa TA, Salanti G, Geddes JR, Higgins JP, Churchill R, Watanabe N, Nakagawa A, Omori IM, McGuire H, et al. Comparative efficacy and acceptability of 12 new-generation antidepressants: a multipletreatments meta-analysis. Lancet. 2009;373(9665):746-58.

21. Liew TM, Lee CS. Comparative efficacy and acceptability of interventions for major depression in older persons: protocol for Bayesian network metaanalysis. BMJ Open. 2018;8(1):e019819.

22. Spiegelhalter DJ, Best NG, Carlin BR, van der Linde A. Bayesian measures of model complexity and fit. J R Stat Soc Series B Stat Methodol. 2002;64:583-616.

23. Zhang Y, Kang S, Fang W, Wu X, Liang W. Network meta-analysis on prophylactic regimens against recurrent hepatitis $B$ virus infection after liver transplantation. Hepatobiliary Surg Nutr. 2013;2(6):297-303.

24. Guyatt G, Oxman AD, Akl EA, Kunz R, Vist G, Brozek J, Norris S, Falck-Ytter Y, Glasziou P, DeBeer H, et al. GRADE guidelines: 1. Introduction-GRADE evidence profiles and summary of findings tables. J Clin Epidemiol. 2011; 64(4):383-94.

25. Singh S, Kumar V, Kumar S, Subbappa A. The effect of periodontal therapy on the improvement of glycemic control in patients with type 2 diabetes mellitus: a randomized controlled clinical trial. Int J Diabetes Dev Ctries. 2008;28(2):38-44.

26. Gilowski L, Kondzielnik P, Wiench R, Plocica I, Strojek K, Krzeminski TF. Efficacy of short-term adjunctive subantimicrobial dose doxycycline in diabetic patients - randomized study. Oral Dis. 2012;18(8):763-70.

27. Moeintaghavi A, Arab HR, Bozorgnia Y, Kianoush K, Alizadeh M. Non-surgical periodontal therapy affects metabolic control in diabetics: a randomized controlled clinical trial. Aust Dent J. 2012;57:31-7.

28. Gaikwad SP, Gurav AN, Shete AR, Desarda HM. Effect of scaling and root planing combined with systemic doxycycline therapy on glycemic control in diabetes mellitus subjects with chronic generalized periodontitis: a clinical study. J Periodontal Implant Sci. 2013;43:79-86.

29. Pradeep AR, Rao NS, Bajaj P, Kumari M. Efficacy of subgingivally delivered simvastatin in the treatment of patients with type 2 diabetes and chronic periodontitis: a randomized double-masked controlled clinical trial. J Periodontol. 2013;84:24-31.

30. Santos VR, Lima JA, Miranda TS, Gonçalves TE, Figueiredo LC, Faveri M, Duarte PM. Full-mouth disinfection as a therapeutic protocol for type-2 diabetic subjects with chronic periodontitis: twelve-month clinical outcomes: a randomized controlled clinical trial. J Clin Periodontol. 2013;40:155-62

31. Telgi RL, Tandon V, Tangade PS, Tirth A, Kumar S, Yadav V. Efficacy of nonsurgical periodontal therapy on glycaemic control in type II diabetic patients: a randomized controlled clinical trial. J Periodontal Implant Sci. 2013;43(4):177-82

32. Miranda TS, Feres M, Perez-Chaparro PJ, Faveri M, Figueiredo LC, Tamashiro NS, Bastos MF, Duarte PM. Metronidazole and amoxicillin as adjuncts to scaling and root planing for the treatment of type 2 diabetic subjects with periodontitis: 1-year outcomes of a randomized placebo-controlled clinical trial. J Clin Periodontol. 2014;41:890-9.

33. Tsalikis L, Sakellari D, Dagalis $P$, Boura $P$, Konstantinidis A. Effects of doxycycline on clinical, microbiological and immunological parameters in well-controlled diabetes type-2 patients with periodontal disease: a randomized, controlled clinical trial. J Clin Periodontol. 2014;41:972-80.

34. Wu Y, Chen L, Wei B, Luo K, Yan F. Effect of non-surgical periodontal treatment on visfatin concentrations in serum and gingival crevicular fluid of patients with chronic periodontitis and type 2 diabetes mellitus. J Periodontol. 2015;86:795-800.

35. Kocak E, Saglam M, Kayis SA, Dundar N, Kebapcilar L, Loos BG, Hakki SS. Nonsurgical periodontal therapy with/without diode laser modulates metabolic control of type 2 diabetics with periodontitis: a randomized clinical trial. Lasers Med Sci. 2016;31(2):343-53.

36. Ramos UD, Ayub LG, Reino DM, Grisi MF, Taba M, Souza SL, Palioto DB, Novaes AB. Antimicrobial photodynamic therapy as an alternative to systemic antibiotics: results from a double-blind, randomized, placebocontrolled, clinical study on type 2 diabetics. J Clin Periodontol. 2016; 43:147-55.

37. Perez CM, Munoz F, Andriankaja OM, Ritchie CS, Martinez S, Vergara J, Vivaldi J, Lopez L, Campos M, Joshipura KJ. Cross-sectional associations of impaired glucose metabolism measures with bleeding on probing and periodontitis. J Clin Periodontol. 2017;44(2):142-9.

38. Liew AK, Punnanithinont N, Lee YC, Yang J. Effect of non-surgical periodontal treatment on $\mathrm{HbA1c}$ : a meta-analysis of randomized controlled trials. Aust Dent J. 2013;58(3):350-7.

39. Fredeking TM, Zavala-Castro JE, Gonzalez-Martinez P, Moguel-Rodriguez W, Sanchez EC, Foster MJ, Diaz-Quijano FA. Dengue patients treated with doxycycline showed lower mortality associated to a reduction in IL-6 and TNF levels. Recent Pat Antiinfect Drug Discov. 2015;10(1):51-8.

40. Preshaw PM, Alba AL, Herrera D, Jepsen S, Konstantinidis A, Makrilakis K, Taylor R. Periodontitis and diabetes: a two-way relationship. Diabetologia. 2012;55(1):21-31.

41. Sgolastra F, Severino M, Pietropaoli D, Gatto R, Monaco A. Effectiveness of periodontal treatment to improve metabolic control in patients with chronic periodontitis and type 2 diabetes: a meta-analysis of randomized clinical trials. J Periodontol. 2013;84(7):958-73.

42. Teshome A, Yitayeh A. The effect of periodontal therapy on glycemic control and fasting plasma glucose level in type 2 diabetic patients: systematic review and meta-analysis. BMC Oral Health. 2016;17(1):31.

\section{Publisher's Note}

Springer Nature remains neutral with regard to jurisdictional claims in published maps and institutional affiliations.
Ready to submit your research? Choose BMC and benefit from:
- fast, convenient online submission
- thorough peer review by experienced researchers in your field
- rapid publication on acceptance
- support for research data, including large and complex data types
- gold Open Access which fosters wider collaboration and increased citations
- maximum visibility for your research: over $100 \mathrm{M}$ website views per year
At BMC, research is always in progress.
Learn more biomedcentral.com/submissions 